\title{
Calculation Method to Determine the Zone of Underground Damage in Water Supply System Pipelines
}

\author{
Abdulkhakim Salokhiddinov ${ }^{1}$, Andrei Savitsky ${ }^{2}$, Olga Ashirova ${ }^{3,{ }^{*}}$, Poshoazimkhon Khakimova ${ }^{1}$, \\ Mohammad Qaasim Yahyah ${ }^{4}$, Lola Gandjaeva ${ }^{5}$ \\ ${ }^{1}$ Tashkent Institute of Irrigation and Agricultural Mechanization Engineers, Department of Water Resources and Environmental \\ Management, Uzbekistan \\ ${ }^{2 " U z G I P " ~ R e s e a r c h ~ a n d ~ D e s i g n ~ I n s t i t u t e, ~ D e p a r t m e n t ~ o f ~ S t r a t e g i c ~ D e s i g n i n g, ~ U z b e k i s t a n ~}$ \\ ${ }^{3}$ Irrigation and Water Problems Research Center, Water-saving Technologies Laboratory, Uzbekistan \\ ${ }^{4}$ Department of Water Engineering and Hydraulic Structures, Balkh University, Afghanistan \\ ${ }^{5}$ Department of Natural Sciences, Khorezm Mamun Academy, Uzbekistan
}

Received May 8, 2021; Revised August 14, 2021; Accepted September 8, 2021

\section{Cite This Paper in the following Citation Styles}

(a): [1] Abdulkhakim Salokhiddinov, Andrei Savitsky, Olga Ashirova, Poshoazimkhon Khakimova, Mohammad Qaasim Yahyah, Lola Gandjaeva, "Calculation Method to Determine the Zone of Underground Damage in Water Supply System Pipelines," Civil Engineering and Architecture, Vol. 9, No. 6, pp. 1805-1811, 2021. DOI: 10.13189/cea.2021.090614.

(b): Abdulkhakim Salokhiddinov, Andrei Savitsky, Olga Ashirova, Poshoazimkhon Khakimova, Mohammad Qaasim Yahyah, Lola Gandjaeva (2021). Calculation Method to Determine the Zone of Underground Damage in Water Supply System Pipelines. Civil Engineering and Architecture, 9(6), 1805-1811. DOI: 10.13189/cea.2021.090614.

Copyright $\mathrm{C} 2021$ by authors, all rights reserved. Authors agree that this article remains permanently open access under the terms of the Creative Commons Attribution License 4.0 International License

\begin{abstract}
The application of water conservation and leakage prevention at all stages of water delivery to the consumer can provide the rational and efficient use of water resources. The long length of heating and water supply networks, their high degree of wear and tear lead to the appearance of leaks. The paper describes research results on developing the calculating method for detecting underground damage zones in the water supply system pipeline. The basis for this study is the main difference between the regulated water intake for the needs of the water user and the uncontrolled loss of water from a damaged pipeline. The water user takes water from the water supply system in accordance with the designed parameters, and such a water intake will not cause unusual changes in the water pressure in the pipeline. The volume of water lost through pipeline damage will always be proportional to the sharp water pressure change in the pipeline. We developed the mathematical model to substantiate the detecting method of the pipeline damage zone. The model considers the operational characteristics of pipelines to the full extend: head and water flow, the degree of deterioration of water conduit, the geodetic position of water intake points. The mathematical model, confirmed earlier by dozens of independent experiments,
\end{abstract}

made it possible to replace expensive hydraulic experiments and prove the possibility of using the developed method of detecting hidden damages of water conduits by extrapolating the measured piezometric heads. The proposed software system for calculating the underground pipeline damage zone in water supply systems makes it possible to determine the pipeline damage hidden zones based on solving the hydraulic problem for a linear pipeline and studying hydraulic processes. We can use the created optimization model as a proven analog of a physical hydraulic stand in hydraulic studies in the simulation mode.

Keywords Model, Pipeline, Water Supply System, Water Intake, Hidden Zones, Leakage, Water, Pressure

\section{Introduction}

Water in zones of arid climate (to which the Aral Sea basin belongs) is a fundamental resource for the life support of man and society, for the production and economic activities, and the development of the state's 
economy. This is clearly evidenced by the practical experience of using water resources in the Central Asian region over the past 40-45 years, showing that the population of the lower reaches of the Syrdarya and the Amudarya Rivers often suffered from a lack of water and its socio-economic consequences [1].

Deterioration of water supply networks is that in many settlements is one of the main contemporary challenges. Analysis of the state of water supply networks in cities and towns shows that their average wear is up to $42 \%$ $[2,3]$. The appearance of through holes in the walls of the pipelines leads to large losses of transported water, for example, over $13 \mathrm{~m} 3$ of water per day is lost only through a hole with a diameter of $3 \mathrm{~mm}$ at a water pressure in the pipeline of $5 \mathrm{~kg} / \mathrm{cm} 2$. Reducing losses from leaks is the most important measure for providing the population with fresh water. Fast detection and, accordingly, fast elimination of the leak mean additional water delivered to the population $[4,5]$.

We carried studies out to substantiate a new method for determining the zone of underground damage to the pipeline in water supply systems. This method is based on the fundamental difference between controlled water intake by water consumers and uncontrolled water flow through the damaged pipelines. In the first case, consumers use water as a useful and often paid resource, and therefore this water intake from the pipeline most often weakly depends on the pressure in the pipe. With an uncontrolled outflow of water, the relation between the water pressure in the pipe and the rate of its outflow through the damage to the pipeline should be traced.

The result of the difference in the efficiently controlled water intake and the useless loss of water through the damaged pipeline is expressed in the appearance of a fracture in the line of piezometric heads at any and different water pressures at the pipeline inlet. When connecting the measured piezometric heads with lines, it is possible to determine the breaking point on the line of piezometric heads. The breaking point will correspond to the point at which water loss is proportional to pressure change, or in other words, to the point of pipeline damage. The theoretical part of the article is devoted to the hydraulic calculation of the pipeline, demonstrating that the fracture of piezometric lines in the zone of pipeline damage is a constant pattern provided by the laws of fluid flow.

Analysis of the simplest hydraulic equations' analytical solution and an analysis of the solution to the same problem conducted using an optimization model developed by the authors are solved in the simulation mode (all pipeline parameters are fixed and set) instead of a complex study on physical models. The running time of this optimization model in the simulation mode ranges from several seconds to several minutes. We wrote the program in the GAMS language [6, 7].

\section{Materials and Methods}

Currently, there are many methods to detect leaks.

Hydraulic methods: visual control of the water level in hydrants when closing a section of the network; analysis of water losses (detection and determination of the leakage rate on the external network of the city and internal networks of buildings) with the "Seba Dinatronic" Co. laboratory. A significant drawback of this method is the need to disconnect sections of the network, making this method non-operational and time-consuming. In particular, in the case of reinforcement skip, the installation of bafflers on pipelines is required $[8,9,10,11]$. Using this method requires violating the integrity of the pipelines in advance by organizing special windows in the pipeline for control and using reinforced concrete pipes to make breaks in the fittings, violating the strength characteristics of the pipelines.

Acoustic methods: acoustic; correlation-acoustic; leak detection using a push-through microphone SOK A-10; application of leak noise recorders (water supply network monitoring systems). The major disadvantage of the acoustic method is the detection of all the noises, and sometimes it is difficult to distinguish the leakage noise from the extraneous noise $[8,9,12,13,14,15]$.

Other methods: detection of the point of pipeline damage using television diagnostic devices - robotic systems or industrial endoscopes (when recording the water pressure); inspection of water supply networks for leaks using an indicator gas (helium, a mixture of hydrogen and nitrogen); network monitoring using the Sebalog and Zonescan systems [8,9, 16,17, 18, 19, 22].

These methods make it possible to find hidden leaks and areas of underground pipeline damage in water supply systems. However, methods that accurately and effectively determine water losses over a wide section of the network without shutting it down have not been developed enough.

The proposed method for detecting a leakage zone in a pipeline uses the main difference between an uncontrolled leak and a man-controlled water intake from the water supply system. In an uncontrolled leak, the flow rate is proportional to the water pressure in the system. Man-controlled water intake is determined by his needs, which is not a rate but a volume of water. This difference is used to determine the leak area, which may be outside the visual control zone.

\section{Results and Discussion}

Consider a system of equations reflecting changes in hydraulic characteristics over a pipe section of the length $L$.

$$
\begin{gathered}
\frac{d H(x)}{d x}=-F\left\{d_{0}, Q(x)\right\} \\
\frac{d Q(x)}{d x}=-Q_{u}(x)-B(x) \cdot L_{u}
\end{gathered}
$$




$$
H(x)=H_{0} \text { for } x=0
$$

where $H(x)$ - is the head, $\mathrm{m}$;

$x$ - horizontal coordinate, $\mathrm{m}$;

$d_{0}$ - pipeline diameter, $\mathrm{m}$;

$Q(x)$ - water consumption at a given point of the pipeline, $1 / \mathrm{s}$

$F\left\{d_{0}, Q(x)\right\}$ - empirical functions found by scientists as a result of research in hydraulics, $1 / \mathrm{s}$;

$Q_{u}(x)$ - known water intake by consumers per one meter of the pipeline length, $1 / \mathrm{s}$;

$L_{u}$ - water leakage from damage in the pipe at some section of the pipeline length, $1 / \mathrm{s}$;

$B(x)$ - the dimensionless function equal to zero throughout the pipe, except for a small zone in which there is a leak from the pipe. In the area of pipe leakage, this function is one.

Despite the fact that the function $F\left\{d_{0}, Q(x)\right\}$ is nonlinear, we can offer its linear approximation for our problem [20]:

$$
\frac{d H(x)}{d x}=-A\left(d_{0}\right) \cdot Q(x)
$$

Let us differentiate it again along the $x$-axis:

$$
\frac{d^{2} H(x)}{(d x)^{2}}=-A\left(d_{0}\right) \cdot \frac{d Q(x)}{d x}
$$

Substituting the above water balance formula (2) into equation (4), we obtain:

$$
\frac{d^{2} H(x)}{(d x)^{2}}=A\left(d_{0}\right) \cdot\left\{Q_{u}(x)+\frac{B(x)}{A\left(d_{0}\right)} \cdot L_{u}\right\}
$$

What is the difference between controlled useful water intake by the consumer and uncontrolled leakage through damage in the pipe? The consumer determines the water intake with the volume of water he needs. Leakage through damage in the pipe is uncontrolled in volume and proportional to the water pressure in the pipeline. The soil surrounding the pipe can of course form some obstruction to the leakage flow, but it does not change the principle of it. i.e.

$$
L_{u}=k \cdot H(x)
$$

where $k-$ is the coefficient of proportionality, depending on the amount of damage to the integrity of the pipe.

Then

$$
\frac{d^{2} H(x)}{(d x)^{2}}=A\left(d_{0}\right) \cdot\left\{Q_{u}(x)+\frac{B(x)}{A\left(d_{0}\right)} \cdot k \cdot H(x)\right\}
$$

or

$$
\frac{d^{2} H(x)}{(d x)^{2}}=B(x) \cdot k \cdot H(x)+A\left(d_{0}\right) \cdot Q_{u}(x)
$$

It is rather difficult to solve this second-order inhomogeneous differential equation for the arbitrary function $Q_{u}(x)$.

By means of a complicated heuristic approximation of function $Q_{u}(x)$, it is possible to obtain such a form of this equation, which could be solved analytically. However, no need to do it either. We should determine the location of the leak, not the function of the pressure drop in the pipeline.

Suppose that the leaking section is much shorter than the pipe under study.

$$
U \prec<L
$$

where $L-$ is the length of the pipe section under study, m;

$U$ - is the section of leakage from the pipe, $\mathrm{m}$.

Let the leak be located at point $L_{u}$. The pipeline is $L$ long. The beginning of the pipeline section is located at the origin of $x$ coordinate.

Let us find the solution to the equation on the sections $\{0\} \leftarrow \rightarrow\left\{L_{u}\right\}$ and $\left\{\left(L_{u}+\mathrm{U}\right)\right\} \leftarrow \rightarrow\{L\}$.

The main point is that the form of the equation being solved changes:

$$
\frac{d^{2} H(x)}{(d x)^{2}}=A\left(d_{0}\right) \cdot Q_{u}(x)
$$

The value of equals to one at the leakage site only. In other points it is $B(x)=0$.

In general, the solution looks like this:

$$
H(x)=\iint A\left(d_{0}\right) \cdot Q_{u}(x) d x+C_{1} \cdot X+C_{2}
$$

where $C_{I}$ and $C_{2}$ - are the constants of integration that can be found from the boundary conditions.

In the most general case, consistent with practice, the solution will represent a concave line.

If we consider a section of the pipe in which the transit flow rate is much less than the water intake by consumers or the time of the day is chosen when the withdrawal of water by consumers is small or the consumers of water at the time of the study temporarily and significantly reduced the water intake, then the solution for the sections $\{0\} \hookleftarrow\left\{L_{u}\right\}$ and $\left\{\left(L_{u}+U\right)\right\} \leftrightarrow\{L\}$ takes a form of a linear equation:

$$
H(x)=C_{1} \cdot X+C_{2}
$$

Let us assume that there is no water withdrawal at the site of leakage, or it is much less than uncontrolled losses. The higher the flow rate, the greater the drop in pressure, which means that the value of $C_{l}$ is negative, and the value of $C_{2}$ is equal to the head at the entrance to the investigated area. In the area after the leakage zone, the value of $C_{1}$ is also negative, and the value of $C_{2}$ is determined by the following equality:

$$
\mathrm{C}_{1} \cdot X+C_{2}=\left.H_{\mathrm{B}}()\right|_{x=L}
$$

where $\left.H_{b}(x)\right|_{x=L}-$ is the head at the end of the investigated pipe section.

The main thing is that the coefficient $C_{l}$ at the beginning of the area under study is unconditionally less than the coefficient $C_{l}$ at the end of the area under study. Let us prove it.

Let us solve the equation for the section associated with the leak from the pipeline. It is assumed that there is no regulated water intake from a real consumer in the leakage zone. This is quite acceptable and is a consequence of the previously accepted assumption about the point nature of the zone of water leakage from the pipeline. Then from 
equation (8) we get:

$$
\frac{d^{2} H(x)}{(d x)^{2}}-k \cdot H(x)=0
$$

since $B(x)=1$ on the entire site $U$.

The characteristic equation for expression (14) looks as follows:

$$
Y^{2}=k, k>0
$$

Characteristic equation (15) has two real roots:

$$
\lambda_{1,2}=+\sqrt{k},-\sqrt{k}
$$

Then the solution to the equation on site $U$ takes the following form:

$$
H(x)=C_{3} \cdot e^{-\sqrt{k} x}+C_{4} \cdot e^{\sqrt{k} x}
$$

Since the behavior of function $H(x)$ when taking into account the root $+\sqrt{k}$ does not correspond to the physics of the process, then this root of the equation should not be considered, which means $C_{4}=0$.

Let us use the property of function $e^{-\sqrt{k} x}$. The derivative of this function is always negative and decreases in absolute value. This means that the righthand point of the function always has a smaller value than its point located to the left. Consequently, in the zone of water leakage from the pipeline, there will be an abrupt exponential drop in pressure. All three solutions of equation (8) must represent one line on the graph $H(x)$ abscissa, $x$ - ordinate. There will be two discontinuities of the first kind on the line (discontinuity of the first kind - the values of the functions to the left and to the right of the discontinuity are equal, but the derivatives are not equal).

It cannot be otherwise since the pressure inside the pipeline is a continuous function, no matter how insignificant the zone $U$ is. Before and after a leak, the solution presents a slightly curved line (small controlled water intake), in the case of a temporary interruption of controlled withdrawal, the solutions present straight lines. They will necessarily intersect in the area of uncontrolled water leakage.

Consequently, the leakage zone is easy to determine if we plot a straight line at the beginning of the pipeline at an inclination, which corresponds to the solution or measurements, and a line at the end of the investigated section of the pipeline, at an inclination, which also corresponds to the solution or measurements, but at the beginning of the section. The point of intersection will be the point where water losses occur (uncontrolled by man, but proportional to the pressure).

The greater the difference in the inclinations of the lines, the more accurately the point of water loss is determined.

The inclinations of the lines are determined by the curvature of the solution function in the area of water losses and as a result of the conjugation of all three solutions.

If the lines run parallel or almost parallel, then there is no loss in the studied section of the pipeline. Ideally, if there are no leaks or water intakes, the straight lines should coincide.

Within the framework of this scientific research, a hydraulic model was developed and algorithmized, which adequately reflects the real hydraulic processes in pipelines. The adequacy and representativeness of the model was tested by comparing with real hydraulic systems and with the results of calculations using other mathematical models and systems. The operating mode of the model for the studies conducted to substantiate the method for determining the zone of water leakage is a simulation (with given lengths of the pipelines and their diameters). The optimization mode in the model was not used and the optimal choice of diameters was not made, although such a possibility is provided in the model [7] and the corresponding numerical experiments and comparisons proved the correctness of the model in all possible modes of its operation.

However, to conduct a numerical experiment, the model for searching for the optimal parameters of the water supply network, developed by the authors of this article should include an equation linking water withdrawal at some predetermined point (determined leakage location) not with the requirements for water, as in the basic model [21], but with the water withdrawal, proportional to the water pressure at a given point.

Let us take a section of the pipeline $6 \mathrm{~km}$ long, with constant pipe diameter of 10 centimeters and locate the leakage zone at a distance of $2 \mathrm{~km}$ from the beginning of the section (Figure 1). The point of the leakage location is chosen arbitrarily, as well as the length of the pipeline section and the diameter of the pipes; and the task of finding this point will be completed if we can determine it based on the analysis of the solution obtained. 


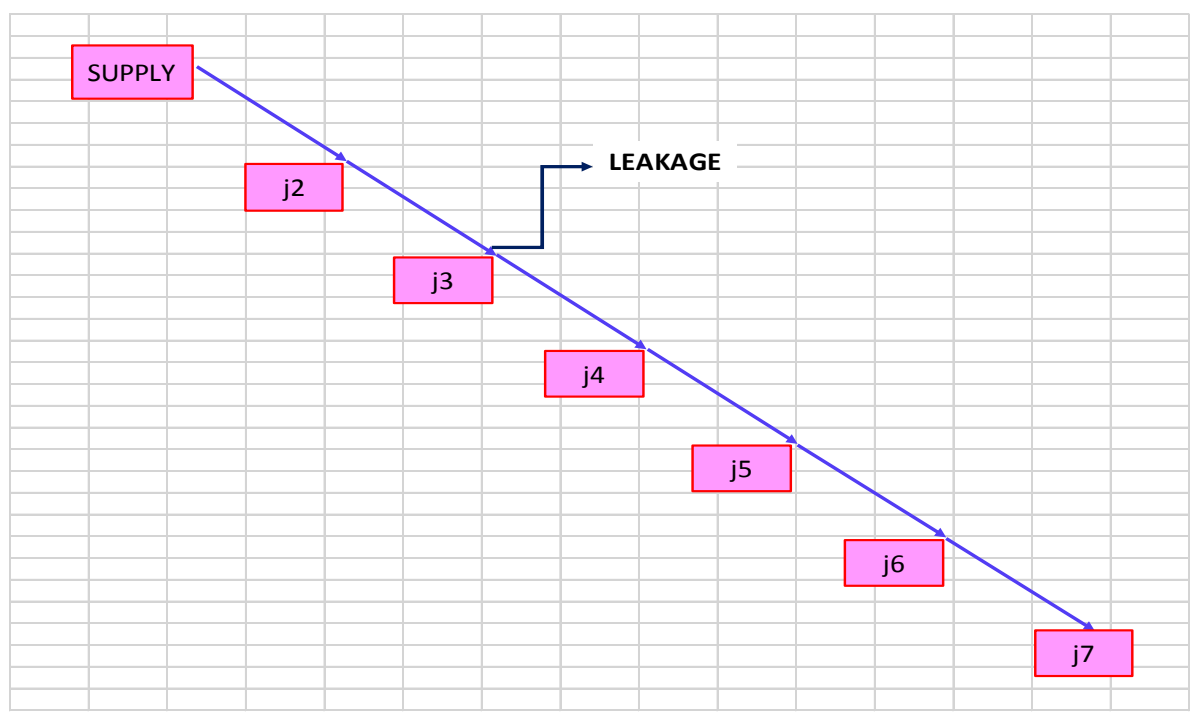

Figure 1. Scheme of a section with an organized leak at node " $\mathrm{j} 3$ " (the distance be-tween nodes is $1 \mathrm{~km}$ )

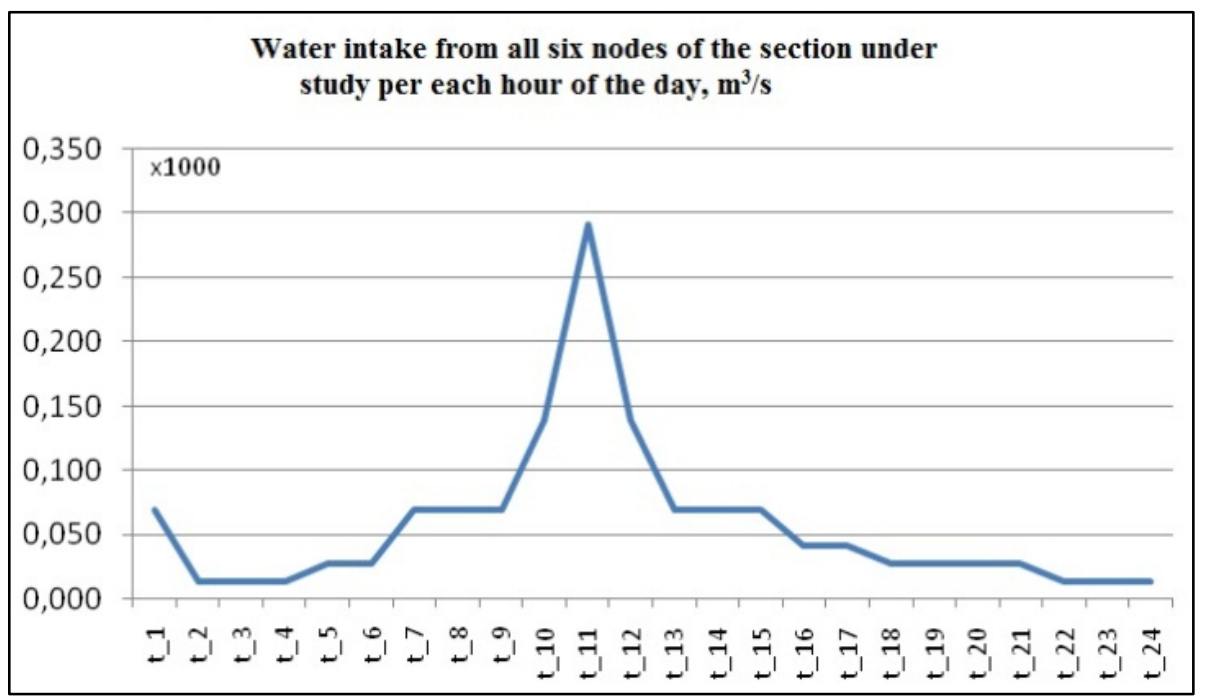

Figure 2. Water intake from all six nodes of the section under study per each hour of the day, $\mathrm{m}^{3} / \mathrm{s}$

Let us assume that on a given $6 \mathrm{~km}$ straight-line section of the network there are water consumers with a predetermined water consumption regime (Figure 2).

Figure 3 shows the solution obtained by the developed optimization model [21] in the simulation mode. The point of intersection of the lines characterizing the drop in pressure at the beginning and end of the investigated section is clearly visible. At the eleventh hour (maximum regulated water withdrawal), the concavity of the pressure drop line is traced, as predicted by the theory set out above. The calculations were performed at different water pressures in the pipeline. The heads were selected in the range from small values, sufficient for the water flow in the pipeline from consumer to consumer, to extremely high values (60 meters of water column). The break in the pressure drop line along the pipeline is most clearly seen at the limiting values of water pressure at the inlet to the pipeline. This is quite understandable since the more noticeable the uncontrolled water leakage from the damage to the pipeline, the higher the water pressure in the pipeline.

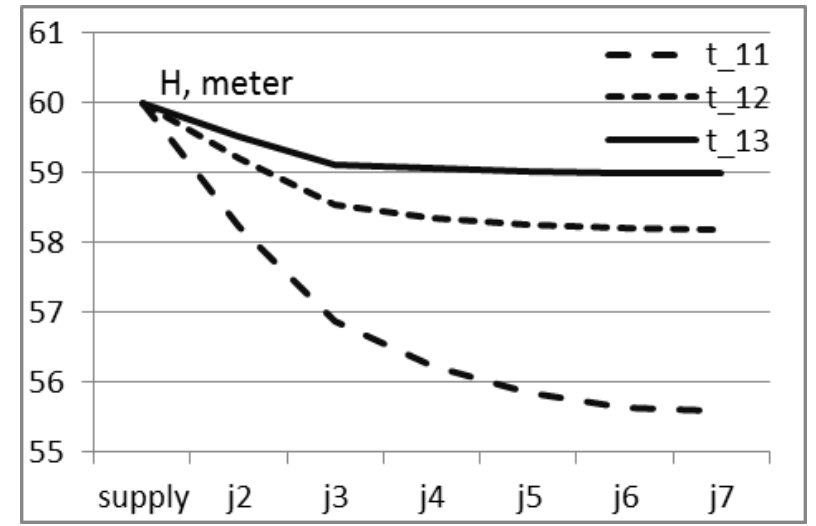

Figure 3. Drop in pressure along the length of the pipe section for three different hours during the day (leakage at point "j_3") 
Figure 3 shows the break in the solution lines in the region of the $\mathrm{j}_{-} 3$ node. A series of real measurements made at the beginning and end of the section of the water supply network should show the same pattern. Extrapolation of piezometric inclinations at the beginning and end of the net-work section must also intersect at point " $j_{-} 3$ ". The model [21] shows the values that real measurements on a real network show since the adequacy and accuracy of the model constructed by the authors and presented in were tested in dozens of independent studies and experiments.

If the leak in the considered problem was at the point $" \mathrm{j}$ $5 "$, then the pressure loss curves would have a different location pattern, and the solution branches to the right and left of the leak point would intersect at the point "j 5 " (Figure 4). A numerical experiment also confirmed this assumption.

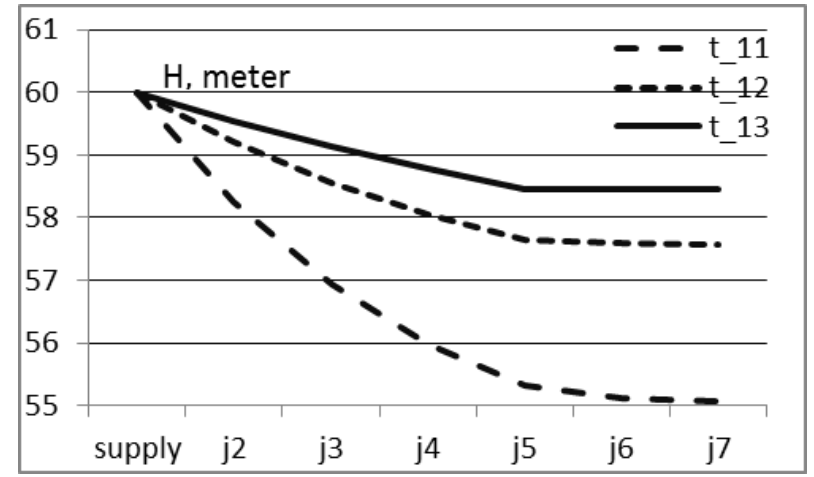

Figure 4. Example of the cycle degeneration to a single point at the prohibition of the formation of a zero link of initial and final points

The main advantage of this method is the accuracy and simplicity to measure the pressure in the pipeline under study. The pressure gauge should be connected to a drain for any two consumers at the end and beginning of the investigated section of the pipeline. It is necessary to accurately determine (using the level) the height of the pressure gauge placement during measurements and the distance between them along the length of the pipe-line. The accuracy can reach several centimeters and millimeters. Within the framework of this study, the development of a method for determining the leakage rate from pipelines was not expected. However, it is quite obvious that it can be defined in this way. In the case of no leak, but damage to the pipeline due to the occurrence of local losses (silting, corrosion, narrowing the free cross-section of the pipeline, compression of the pipeline), the pressure loss lines extrapolated from the beginning and the end of the pipelines are almost parallel; in practice and in theory they simply do not intersect.

\section{Conclusions}

1. The main feature of the controlled water intake by the consumer is a less noticeable dependence on the water pressure in the pipeline. At high and normal pressures the consumer intakes the water in volumes within the designed parameters. Water loss due to pipeline damage has a higher dependence on the water pressure sharp change in the pipeline. This fundamental difference between the useful and useless water flow out of the pipeline allowed us to assume the existence of some feature on piezometric lines at different water pressures in the pipeline.

2. The hydraulic problem under study included a mathematical description of a fixed, controlled water intake and water losses proportional to a sharp change in water pressure simulating integrity damage of the pipeline. The system of equations was solved and the results showed the existence of a fracture in the line of piezometric head. Moreover, the higher the pressure, the stronger the fracture of the line of piezometric head.

3. In practice, the area of integrity damage of the pipeline can be determined as follows. For a series of different pressures, the piezometric head is measured at the points where it is available. The lines of piezometric head are constructed. The breaking point in the lines in a certain area is an indicator of the existence of pipeline damage in this zone.

4. When measuring piezometric heads, it is recommended to provide a temporary increased pressure and, if possible, reduce the water intake by consumers. In this case, the fracture zone of the line of piezometric head can be determined with higher accuracy.

\section{REFERENCES}

[1] National report on the state of the environment and the use of natural resources in the Republic of Uzbekistan (20082011). Tashkent, 2013.

[2] Improving urban management and urban infrastructure in Uzbekistan: problems and the search for new mechanisms and tools: analytical report. Tashkent: Center for Economic Research, 2011.

[3] A. Salokhiddinov, A. Savitsky, O. Ashirova, P. Khakimova, A. Khomidov. An Analytical Method for Designing of Municipal Water Supply and Distribution Systems. Civil Engineering and Architecture Vol. 8(3), pp. $200-205$. DOI:10.13189/cea.2020.080302

[4] O. Ashirova, A. Salokhiddinov. Effective hidden water leaks detecting method in underground pipelines. Scientific Journal of Russian Scientific Research Institute of Land Improvement Problems. № 2(26), 2017.

[5] O. Primin. Ways of Reducing Water Loss. Communal complex of Russia. No. 3 (201), 2021.

[6] Bruce A. McCarl et al. Expanded GAMS User Guide, GAMS Release 24.2.1. GAMS Development Corporation, 
Washington, DC, USA, 2013.

[7] O. Ashirova, A. Savitsky, A. Salokhiddinov. Calculation program to determine the zone of underground damage to the pipeline in water supply systems. Certificate of state registration of the computer soft-ware No. DGU 03705. Tashkent, 2016.

[8] A. Kosygin, V. Khanin, K. Gosudarev, I. Fomina. Detection of hidden leaks using a water supply network monitoring system - Water supply and sanitary equipment. 2010. No. 4. pp. 22-26.

[9] A. Kosygin, V. Khanin, K. Gosudarev, I. Fomina. Monitoring of hidden leaks on the water supply network. Report presented at the international conference "NO-DIG-2010". Center for technical diagnostics "Mosvodokanal". Moscow, 2010.

[10] A. Timoshkin. Devices for determining the state and places of damage of pipelines of heating networks. Heat supply news. 2001. No. 2 (06). pp. 29-31.

[11] L. Kublanovsky. Determination of the point of damage of pressure pipelines. - M.: Nedra, 1971.

[12] Y. Khulief, A. Khalifa, R. Ben-Mansour, M. Habib. Acoustic Detection of Leaks in Water Pipelines Using Measurements inside Pipe. Journal of Pipeline Systems Engineering and Practice 3(2):47. DOI: 10.1061/(ASCE)PS.1949-1204.0000089

[13] N. Adnan et al. Leak detection in gas pipeline by acoustic and signal processing - A review. 2015 IOP Conf. Ser.: Mater. Sci. Eng. 100012013

[14] M. Ramírez, L. Borges. Acoustic Water Leak Detection
System. VII International Telecommunications Symposium. 2010. DOI: $10.14209 /$ sbrt.2010.117

[15] D. Kumar, D. Tu, N. Zhu, D. Hou, H. Zhang. In-Line Acoustic Device Inspection of Leakage in Water Distribution Pipes Based on Wavelet and Neural Network. Journal of Sensors, vol. 2017, Article ID 5789510, 2017. https://doi.org/10.1155/2017/5789510

[16] El-Zahab, S., Zayed, T. Leak detection in water distribution networks: an introductory overview. Smart Water 4, 5 (2019). https://doi.org/10.1186/s40713-019-0017-x

[17] Y. Liu, X. Ma, Y. Li, Y. Tie, Y. Zhang, J. Gao. Water Pipeline Leakage Detection Based on Machine Learning and Wireless Sensor Networks Sensors (Basel). 2019 Dec; 19(23): 5086. Published online 2019 Nov 21. doi: $10.3390 / \mathrm{s} 19235086$

[18] D. Covas, H.Ramos. Hydraulic transients used for leak detection in water distribution systems. International Conference in Water Pipeline Systems. 2001.

[19] Water Loss Detectives. Romanian leakage magazine. 2019, year IX / no. 12. www.detectiviiapeipierdute.ro

[20] N. Piskunov. Differential and integral calculus. In 2 volumes. - M.: Fizmatlit, 1972.

[21] Salokhiddinov, A.T. Mathematical model for calculating the water supply network with the possibility of optimizing its elements / A.G. Savit-sky, O.A. Ashirova. - Irrigation and reclamation. - 2015. No. 01. - P. 42-47.

[22] A. Nahwani, A. Husin. Water Network Improvement Using Infrastructure Leakage Index and Geographic Information System. Civil Engineering and Architecture Vol. 9(3), pp. 909 - 914. DOI: 10.13189/cea.2021.090333 